La restitution même partielle d'un tel ensemble qui n'a pas d'équivalent dans un musée espagnol ou au Louvre, montre l'artiste sous un aspect spectaculaire et grandiose, encore ignoré en France où Alonso Cano est presque un inconnu. Avoir sauvé cet ensemble grâce à la ténacité d'un conservateur ${ }^{17}$ et la générosité d'un donateur prouve, s'il en est besoin, le rôle essentiel des musées qui, parfois, peuvent adoucir les vicissitudes du temps. Il demeure significatif que ces merveilleuses acquisitions aient été le point de départ d'une politique d'enrichissement des fonds du Musée Goya qui en fait désormais le sanctuaire de l'Hispanité en France.

JeAn-Louis Augé

Conservateur en Chef des Musées Goya et Jaurès

\title{
NUEVOS DIBUJOS DE ALONSO CANO Y SU CÍRCULO
}

No es fácil abordar el estudio de los dibujos de Alonso Cano '. Su versatilidad, su dominio y aplicación en las diferentes técnicas y sobre todo las obras de su círculo más inmediato y de seguidores y copistas dificultan en grado extremo la catalogación correcta de sus diseños, algo imprescindible para poder conocer en profundidad la obra y el alcance de la pintura del artista granadino, así como su forma de trabajo, que ejemplifica sin lugar a dudas las preocupaciones de un hombre culto, formado en un ambiente intelectual y erudito y sobre todo con un bagaje cultural amplio según se deduce de su biblioteca comprada por Vicente Salvador Gómez en $1673^{2}$ y que se publica en este mismo número de Archivo.

Precisamente tanto en el documento primero de la compra de los bienes de Cano como en el inventario de Vicente Salvador Gómez aparecen dibujos y estampas, elementos muy preciados en la España del siglo XviI para el trabajo de los artistas, pues se convertían en verdaderas canteras para la inspiración en el proceso de elaboración de la obra artística ${ }^{3}$.

El propósito del presente artículo es dar a conocer seis nuevos dibujos debidos algunos a la mano del propio racionero y otros de su círculo más inmediato.

El primer grupo de dibujos viene a completar la serie de dibujos que Cano ejecutó para los lienzos del claustro del Real Convento de Santa Cruz de Granada dedicado a la vida de Santo Domingo e incluyendo algunos hechos franciscanos, para hermanar las dos ordenes mendicantes, que en su inicio tuvieron graves disensiones. El propio Palomino dio noticia de estos dibujos que pertenecían a su propia colección:

«Volvió, pues, a Granada nuestro Racionero, donde ejecutó diferentes pinturas, y esculturas, para algunos amigos, y personas particulares. $Y$ en este tiempo hizo todos los dibujos, para las pinturas del claustro del Real Convento de Santa Cruz,

\footnotetext{
1 Para el estudio de los dibujos de Cano siguen siendo útiles los artículos de H. Wethey en el Art Bulletin, 1952 y en los Estudios sobre literatura y arte dedicados al Profesor Emilio Orozco Díaz, 1979, donde se aceptan y recogen las afirmaciones de A.E. Pérez Sánchez respecto a dibujos de los Uffizi en principio rechazadas. Recientemente véase el completo trabajo de Zahira Véliz, Alonso Cano's Drawings and Related Works, Londres, Courtauld Institute-London University, 1998.

2 Navarrete Prieto, B., «Sobre Vicente Salvador Gómez y Alonso Cano. Nuevos documentos y fuentes formales» en Ars Longa, 1996, pp. 77-82.

3 Véase nuestra tesis doctoral; La Pintura Andaluza del siglo XVII y sus fuentes grabadas, Fundación de Apoyo a la Historia del Arte Hispánico, Madrid, 1998. Especialmente el capítulo dedicado a las bibliotecas e inventarios de artistas como canteras de la inspiración, pp. 63-73.
} 

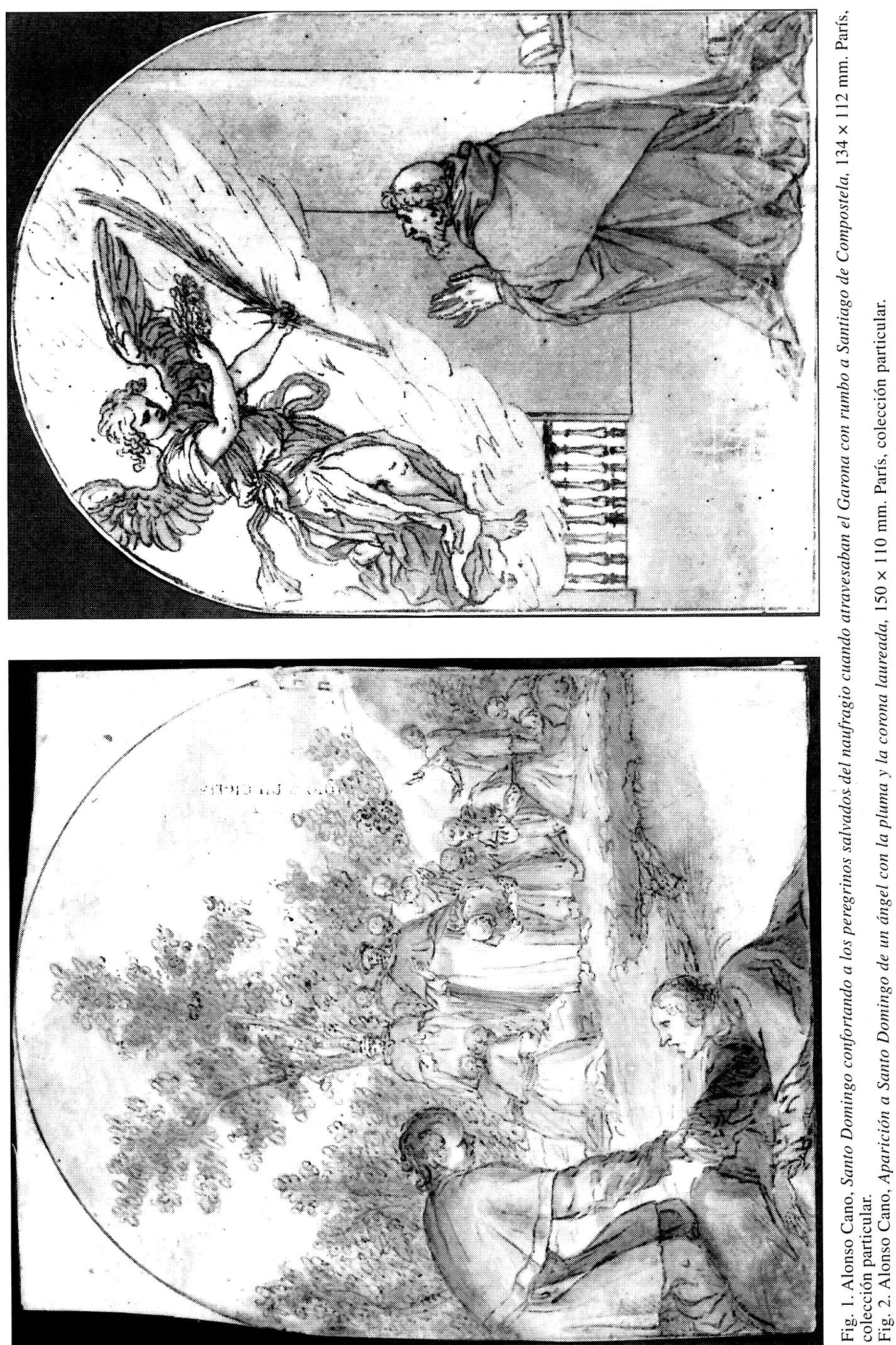
Orden de Predicadores, de la Vida de su glorioso Patriarca, los cuales tengo yo en mi poder. Pero las pinturas en dicho claustro las ejecutó por los dibujos de Cano un fulano del Castillo, y están ya muy deterioradas del tiempo» ${ }^{4}$.

Desde que Sánchez Cantón ${ }^{5}$ recogió siete dibujos relativos a este conjunto se han venido añadiendo otros, sobre todo por Wethey, y han aparecido copias de algunos de los dados a conocer, por lo que se deduce que deben de existir varias series que copiaran la original de Cano de calidad muy desigual como nos informa Zahira Véliz.

Hasta la fecha se tenía constancia de once composiciones en relación con este ciclo dominico recientemente expuestas en el Museo del Prado ${ }^{6}$. Los temas son: Bautismo de Santo Domingo, Santo Domingo niño durmiendo en el suelo, Santo Domingo bendiciendo a los peregrinos rescatados de las aguas, La Virgen entregando el Rosario a Santo Domingo, El sueño del papa Honorio III, Santo Domingo, San Francisco y la Virgen intercediendo por la Humanidad, La cena milagrosa de santo Domingo, Ángeles con espadas de fuego persiguiendo a los herejes, Milagros ante el sepulcro de san Pedro mártir, San Agustín o Santo Domingo ante el papa y Santo Domingo en Soriano.

Ahora podemos añadir dos nuevas composiciones:

- Santo Domingo confortando a los peregrinos salvados del naufragio cuando atravesaban el Garona con rumbo a Santiago de Compostela, $134 \times 112 \mathrm{~mm}$., pluma y tinta, y aguada sepia sobre preparación de grafito (Fig. 1). De formato rectangular muestra el medio punto a pluma como algunos de la serie.

Se trata de una interesante composición, no conocida, que representa de modo muy diferente el mismo tema que conserva el Museo del Prado, procedente de la colección Fernández Durán. La calidad es semejante y la técnica similar pudiendo pensarse en escena complementaria a la anterior por presentar formato similar a los restantes de la serie y diferente al que conserva el Prado, único diseño de esta serie de formato mayor, probablemente para servir como preparatorio para una sobrepuerta.

El dibujo dado ahora a conocer muestra en primer término a un clérigo que rescata de las aguas a un peregrino mientras que Santo Domingo se encuentra al fondo en tierra firme confortando a los demás que han sido salvados de las aguas; al fondo unas arboledas.

- Aparición a Santo Domingo del Ángel con la palma y la corona laureada, $150 \times 110$ $\mathrm{mm}$. Pluma, tinta y aguada sepia (Fig. 2). Recortado en la parte superior y silueteado en forma de medio punto.

Composición también inédita que por su carácter y formato, parece pertenecer a la serie de Santo Domingo y San Francisco. Presenta a un santo implorante en el momento de la aparición de un ángel con la corona de laurel y la palma del martirio envuelto en una aureola llameante.

- Santo Domingo, San Francisco y la Virgen intercediendo por la humanidad, $141 \times 127$ $\mathrm{mm}$. Pluma, tinta y aguada sepia sobre preparación de grafito (Fig. 3). De tamaño rectangular y rematado con pluma el medio punto, el dibujo ha sido recortado

Se trata de versión de la composición conservada en el Museo del Prado, de similar tamaño y con la tinta más diluida. Probablemente se trata de una copia, aunque existen algunas variantes como presentar las tres flechas que sostiene Cristo más separadas.

\footnotetext{
${ }^{4}$ Palomino, A., Museo Pictórico y escala óptica con el parnaso español pintoresco y laureado, ed. Madrid, 1947, p. 992. Antonio García Bascón, Director del Museo de Bellas Artes de Granada, nos informa de que actualmente todavía se conservan en el museo restos de estos lienzos.

5 Sánchez Cantón, F.J., Dibujos Españoles. IV Alonso Cano, Ministerio de Instrucción Pública y Bellas Artes, Madrid, 1930.

6 Véliz, Z., Alonso Cano. Dibujos, cat. exp., Museo del Prado, Madrid, 2001, cat. 57-67.
} 

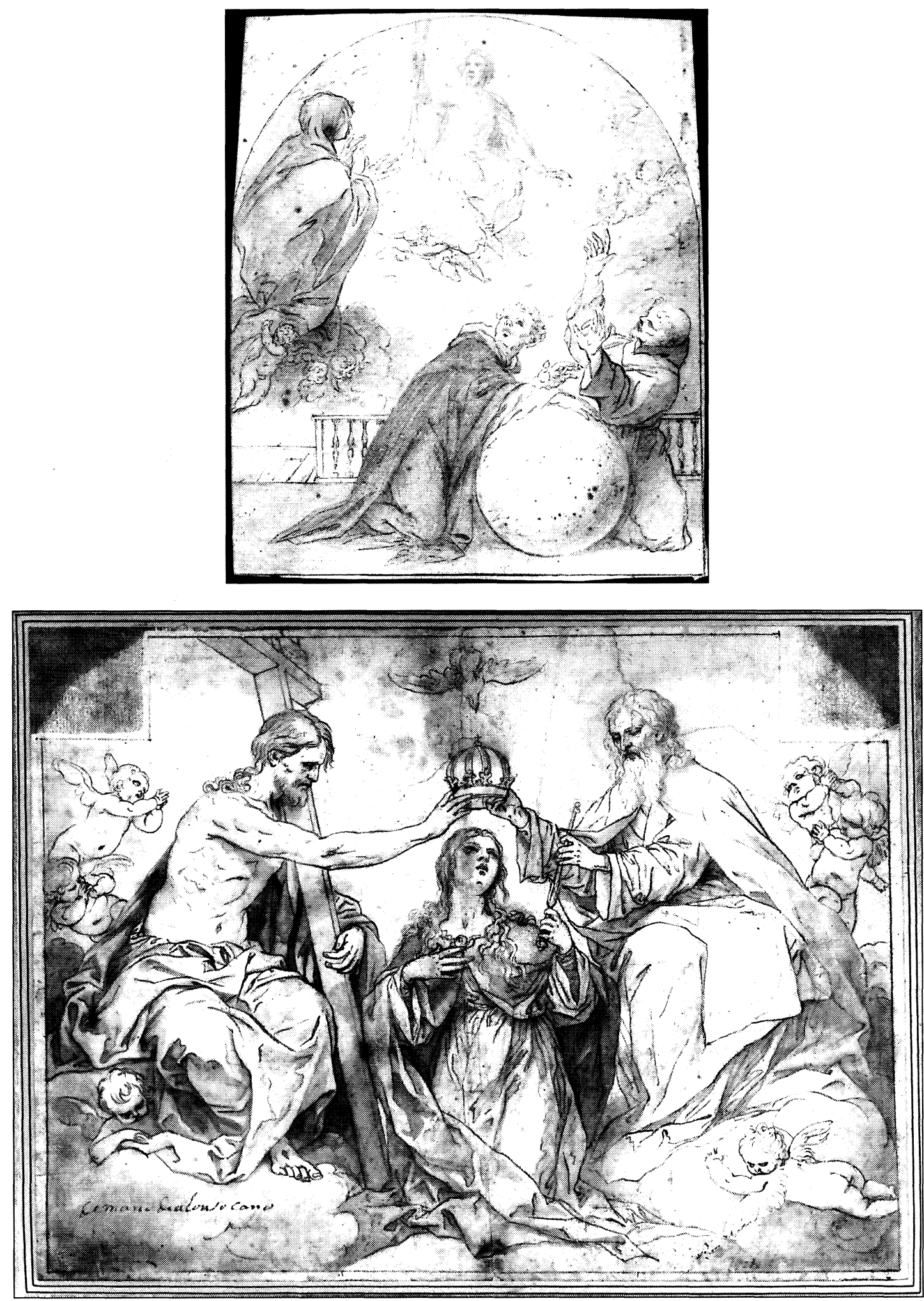

Fig. 3. ¿Copia a partir de una composición de Alonso Cano?, Santo Domingo, San Francisco y la Virgen intercediendo por la humanidad, $141 \times 127 \mathrm{~mm}$. París, colección particular.

Fig. 4. Alonso Cano, Coronación de la Virgen, $227 \times 300 \mathrm{~mm}$. París, colección particular. 
El dibujo hace alusión a la visión de Santo Domingo de Cristo con tres flechas e irritado con la humanidad a la que se disponía a lanzar tres flechas contra el orgullo, la avaricia y la lujuria. La Virgen intercede y muestra abajo a Santo Domingo y San Francisco que velarán por la obediencia, pobreza y castidad ${ }^{7}$.

Independientes de esta serie damos ahora a conocer dos dibujos de propiedad particular uno de mano de Alonso Cano y representando:

- La Coronación de la Virgen, $227 \times 300 \mathrm{~mm}$. Pluma y tinta parda, aguada castaña sobre preparación de grafito, papel amarillento (Fig. 4). Anotación en la parte inferior izquierda: «de mano de alonso cano». Recortados los ángulos superiores.

En relación con el dibujo del mismo asunto conservado en los Uffizi, presenta similar técnica y sobre todo parece ser como aquél dibujo preparatorio para ático de retablo.

El modo de solucionar la figura de Cristo es similar, así como la forma de conformar la cruz.

Sánchez Cantón mencionó el dibujo florentino como probable boceto del cuadro para el ático del retablo en que estaba la Inmaculada de José de Mora en San Isidro de Madrid.

La fecha de ambos diseños debe de ser cercana a 1650.

Finalmente queremos dar a conocer una Muerte de la Magdalena, $900 \times 130 \mathrm{~mm}$. Pluma y tinta sepia (Fig. 5). Estrechamente relacionada en cuanto a técnica y factura con el Cristo de la resurrección y el Joven sentado de los Uffizi apreciando similar violencia en el uso de la pluma y en la grafía alborotada, por lo que puede relacionarse igualmente con el pintor granadino.

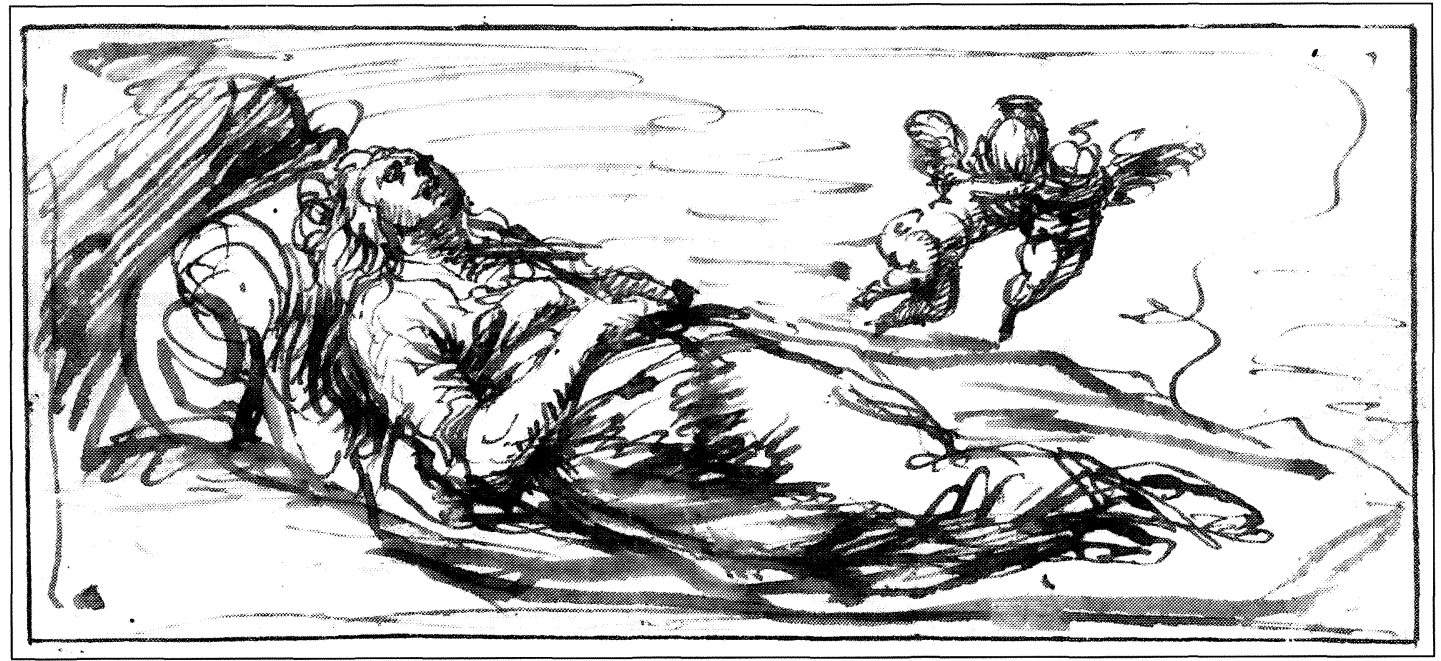

Fig. 5. ¿Alonso Cano?, Muerte de la Magdalena, $900 \times 130$ mm. Colección particular.

Aparece la Magdalena echada, probablemente en el momento de su tránsito, porta en las manos una cruz muy esbozada y a la derecha dos angelotes. Se trata de un interesante diseño en el que se aprecia el audaz uso de la pluma gruesa probablemente de caña.

Estos nuevos dibujos habrán de ser tenidos en cuenta en lo sucesivo en los estudios de Cano y sus seguidores pues los de la serie de Santo Domingo nos transmiten nuevas composiciones, si no de mano del maestro, desde luego testimonio de composiciones perdidas.

Benito Navarrete Prieto Universidad de Valladolid

${ }^{7}$ Reau, L., Iconografía del arte cristiano. Iconografía de los santos, tomo 2, vol. 3, pp. 399-400. 